\title{
Positive solutions for singular nonlinear fractional differential equation with integral boundary conditions
}

\author{
Hongdan $\mathrm{Li}^{1}$, Lishan $\mathrm{Liu}^{1,2^{*}}$ and Yonghong $\mathrm{Wu}^{2}$
}

\author{
"Correspondence: \\ mathlls@163.com \\ 'School of Mathematical Sciences, \\ Qufu Normal University, Qufu, \\ Shandong 273165, People's \\ Republic of China \\ ${ }^{2}$ Department of Mathematics and \\ Statistics, Curtin University, Perth, \\ WA 6845, Australia
}

\begin{abstract}
In this article, we study the existence of positive solutions for a class of singular nonlinear fractional differential equations with Riemann-Stieltjes integral boundary conditions. Using the properties of the Green function and the fixed point theory in cones, we obtain some results on the existence of positive solutions. Our results extend and improve many known results including singular and nonsingular cases.
\end{abstract}

MSC: $34 \mathrm{~A} 08 ; 34 \mathrm{~B} 16 ; 34 \mathrm{~B} 18$

Keywords: singular fractional differential equations; Riemann-Stieltjes integral boundary value problem; positive solution; multiple positive solutions; fixed point theorem in cone

\section{Introduction}

In this article, we consider the existence of solutions for the following class of singular fractional differential equations:

$$
\left\{\begin{array}{l}
D_{0^{+}}^{\alpha} u(t)+p(t) f(t, u(t))+q(t) g(t, u(t))=0, \quad 0<t<1, \\
u(0)=u^{\prime}(0)=\cdots=u^{(n-2)}(0)=0, \\
u(1)=\int_{0}^{1} h(s) u(s) d A(s)
\end{array}\right.
$$

where $n-1<\alpha \leq n, n \geq 2, p, q \in C(0,1), p(t)$ and $q(t)$ are allowed to be singular at $t=0$ or $t=1, f, g:[0,1] \times(0, \infty) \rightarrow[0, \infty)$ are continuous and may be singular at $x=0, h:(0,1) \rightarrow$ $[0, \infty)$ is continuous with $h \in L^{1}(0,1)$, and $\int_{0}^{1} h(s) u(s) d A(s)$ denotes the Riemann-Stieltjes integral with a signed measure, in which $A:[0,1] \rightarrow \mathbb{R}$ is a function of bounded variation.

Fractional differential equations have attracted more and more attention from the research communities due to their numerous applications in many fields of science and engineering including fluid flow, rheology, diffusive transport akin to diffusion, electrical networks, probability, etc. For details, see [1-6] and the references therein. On the other hand, boundary value problems with integral boundary conditions for ordinary differential equations arise in many fields of applied mathematics and physics such as heat conduction, chemical engineering, underground water flow, thermoelasticity, and plasma physics. The existence and multiplicity of positive solutions for such problems have become an important area of investigation in recent years.

(c) $2015 \mathrm{Li}$ et al. This article is distributed under the terms of the Creative Commons Attribution 4.0 International License (http://creativecommons.org/licenses/by/4.0/), which permits unrestricted use, distribution, and reproduction in any medium, provided you give appropriate credit to the original author(s) and the source, provide a link to the Creative Commons license, and indicate if changes were made. 
Cabada and Hamdi [7] studied the existence of positive solutions of the following nonlinear fractional differential equation with integral boundary value conditions:

$$
\left\{\begin{array}{l}
D_{0^{+}}^{\alpha} u(t)+f(t, u(t))=0, \quad 0<t<1 \\
u(0)=u^{\prime}(0)=0, \quad u(1)=\lambda \int_{0}^{1} u(s) d s
\end{array}\right.
$$

where $2<\alpha \leq 3,0<\lambda, \lambda \neq \alpha, D_{0^{+}}^{\alpha}$ is the Riemann-Liouville fractional derivative, and $f$ : $[0,1] \times[0, \infty) \rightarrow[0, \infty)$ is a continuous function.

By means of the monotone iteration method, Sun and Zhao [8] investigated the existence of positive solutions for the fractional differential equation with integral boundary conditions

$$
\left\{\begin{array}{l}
D_{0^{+}}^{\alpha} u(t)+q(t) f(t, u(t))=0, \quad 0<t<1, \\
u(0)=u^{\prime}(0)=0, \quad u(1)=\int_{0}^{1} g(s) u(s) d s,
\end{array}\right.
$$

where $2<\alpha \leq 3, D_{0^{+}}^{\alpha}$ is the standard Riemann-Liouville derivative of order $\alpha, f:[0,1] \times$ $[0, \infty) \rightarrow[0, \infty)$ is continuous, and $g, q:(0,1) \rightarrow[0, \infty)$ are also continuous with $g, q \in$ $L^{1}(0,1)$.

Zhang et al. [9] considered the following nonlinear fractional differential equation with integral boundary conditions:

$$
\begin{cases}D_{0^{+}}^{\alpha} u(t)+h(t) f(t, u(t))=0, & 0<t<1, \\ u(0)=u^{\prime}(0)=u^{\prime \prime}(0)=0, & u(1)=\lambda \int_{0}^{\eta} u(s) d s\end{cases}
$$

where $3<\alpha \leq 4,0<\eta \leq 1,0 \leq \frac{\lambda \eta^{\alpha}}{\alpha}<1, D_{0^{+}}^{\alpha}$ is the Riemann-Liouville fractional derivative, $h:(0,1) \rightarrow[0, \infty)$ is continuous, and $f:[0,1] \times[0, \infty) \rightarrow[0, \infty)$ is also continuous.

Motivated by the above-mentioned papers, the purpose of this article is to investigate the existence of positive solutions for the more general fractional differential equations BVP (1.1) under suitable conditions on $f$ and $g$. Obviously, our discussion is different from that in [7-11]. The main new features presented in this paper are as follows. First, the boundary value problem has a more general form, in which $p, q$ are allowed to be singular at $t=0,1$ and $f, g$ may be singular at $x=0$. Second, the existence and multiplicity of positive solutions of BVP (1.1) are obtained. Third, $\int_{0}^{1} u(s) d A(s)$ denotes the Riemann-Stieltjes integral, where $A$ is a function of bounded variation, and $d A$ may be a signed measure. The work includes the multipoint boundary problems and integral boundary problems as special cases.

The rest of the paper is organized as follows. In Section 2, we present some preliminaries and lemmas that are used to prove our main results. We also develop some properties of the Green function. In Section 3, we discuss the existence of a positive solution of BVP (1.1). In Section 4, we discuss the existence of multiple positive solutions of BVP (1.1). In Section 5, we give an example to demonstrate an application of our theoretical results.

\section{Preliminaries and lemmas}

In this section, for the convenience of reader, we present some notation and lemmas that will be used in the proof of our main results. They can be found in the literature; see $[5,6$, $12,13]$. 
Definition 2.1 The Riemann-Liouville fractional integral of order $\alpha>0$ of a function $y$ : $(0, \infty) \rightarrow \mathbb{R}$ is given by

$$
I_{0^{+}}^{\alpha} y(t)=\frac{1}{\Gamma(\alpha)} \int_{0}^{t}(t-s)^{\alpha-1} y(s) d s
$$

provided that the right-hand side is pointwise defined on $(0, \infty)$.

Definition 2.2 The Riemann-Liouville fractional derivative of order $\alpha>0$ of a continuous function $y:(0, \infty) \rightarrow \mathbb{R}$ is given by

$$
D_{0^{+}}^{\alpha} y(t)=\frac{1}{\Gamma(n-\alpha)}\left(\frac{d}{d t}\right)^{n} \int_{0}^{t} \frac{y(s)}{(t-s)^{\alpha-n+1}} d s
$$

where $n=[\alpha]+1$ with $[\alpha]$ denoting the integer part of a number $\alpha$, provided that the right-hand side is pointwise defined on $(0, \infty)$.

Lemma 2.1 [6] Let $\alpha>0$. If $u \in C(0,1) \cap L^{1}(0,1)$, then the fractional differential equation

$$
D_{0^{+}}^{\alpha} u(t)=0
$$

has

$$
u(t)=C_{1} t^{\alpha-1}+C_{2} t^{\alpha-2}+\cdots+C_{N} t^{\alpha-N}, \quad C_{i} \in \mathbb{R}, i=1,2, \ldots, N,
$$

as the unique solution, where $N=[\alpha]+1$.

From the definition of the Riemann-Liouville derivative we can obtain the following statement.

Lemma 2.2 [6] Assume that $u \in C(0,1) \cap L^{1}(0,1)$ with a fractional derivative of order $\alpha>0$ that belongs to $C(0,1) \cap L^{1}(0,1)$. Then

$$
I_{0^{+}}^{\alpha} D_{0^{+}}^{\alpha} u(t)=u(t)+C_{1} t^{\alpha-1}+C_{2} t^{\alpha-2}+\cdots+C_{N} t^{\alpha-N}
$$

for some $C_{i} \in \mathbb{R}(i=1,2, \ldots, N)$, where $N=[\alpha]+1$.

In the following, we present the Green function of the fractional differential equation boundary value problem.

Lemma 2.3 Given $y \in C(0,1) \cap L^{1}(0,1), n-1<\alpha \leq n$, the problem

$$
\left\{\begin{array}{l}
D_{0^{+}}^{\alpha} u(t)+y(t)=0, \quad 0<t<1, \\
u(0)=u^{\prime}(0)=\cdots=u^{(n-2)}(0)=0, \\
u(1)=\int_{0}^{1} h(s) u(s) d A(s)
\end{array}\right.
$$

is equivalent to

$$
u(t)=\int_{0}^{1} G(t, s) y(s) d s,
$$


where

$$
G(t, s)=G_{0}(t, s)+\frac{t^{\alpha-1}}{1-\Gamma} g_{A}(s)
$$

with

$$
\begin{aligned}
& G_{0}(t, s)=\frac{1}{\Gamma(\alpha)} \begin{cases}{[t(1-s)]^{\alpha-1}-(t-s)^{\alpha-1},} & 0 \leq s \leq t \leq 1, \\
{[t(1-s)]^{\alpha-1},} & 0 \leq t \leq s \leq 1,\end{cases} \\
& \Gamma=\int_{0}^{1} t^{\alpha-1} h(t) d A(t), \quad g_{A}(s)=\int_{0}^{1} G_{0}(t, s) h(t) d A(t) .
\end{aligned}
$$

Proof We may apply Lemma 2.2 to reduce (2.1) to the equivalent integral equation

$$
u(t)=-I_{0^{+}}^{\alpha} y(t)+C_{1} t^{\alpha-1}+C_{2} t^{\alpha-2}+\cdots+C_{n} t^{\alpha-n}
$$

for some $C_{i} \in \mathbb{R}(i=1,2, \ldots, n)$. Consequently, the general solution of (2.1) is

$$
u(t)=-\frac{1}{\Gamma(\alpha)} \int_{0}^{t}(t-s)^{\alpha-1} y(s) d s+C_{1} t^{\alpha-1}+C_{2} t^{\alpha-2}+\cdots+C_{n} t^{\alpha-n}
$$

Since $u(0)=u^{\prime}(0)=\cdots=u^{(n-2)}(0)=0$, we get that $C_{2}=C_{3}=\cdots=C_{n}=0$ by (2.3). Then, we obtain

$$
u(t)=-\frac{1}{\Gamma(\alpha)} \int_{0}^{t}(t-s)^{\alpha-1} y(s) d s+C_{1} t^{\alpha-1}
$$

On the other hand, we can combine (2.1) with

$$
u(1)=-\frac{1}{\Gamma(\alpha)} \int_{0}^{1}(1-s)^{\alpha-1} y(s) d s+C_{1},
$$

yielding

$$
C_{1}=\frac{1}{\Gamma(\alpha)} \int_{0}^{1}(1-s)^{\alpha-1} y(s) d s+\int_{0}^{1} h(s) u(s) d A(s) .
$$

So, by (2.4) and (2.5) we have

$$
\begin{aligned}
u(t)= & -\frac{1}{\Gamma(\alpha)} \int_{0}^{t}(t-s)^{\alpha-1} y(s) d s+\frac{t^{\alpha-1}}{\Gamma(\alpha)} \int_{0}^{1}(1-s)^{\alpha-1} y(s) d s \\
& +t^{\alpha-1} \int_{0}^{1} h(s) u(s) d A(s) \\
= & \frac{1}{\Gamma(\alpha)} \int_{0}^{t}\left[t^{\alpha-1}(1-s)^{\alpha-1}-(t-s)^{\alpha-1}\right] y(s) d s \\
& +\frac{1}{\Gamma(\alpha)} \int_{t}^{1} t^{\alpha-1}(1-s)^{\alpha-1} y(s) d s+t^{\alpha-1} \int_{0}^{1} h(s) u(s) d A(s) .
\end{aligned}
$$


Let

$$
G_{0}(t, s)=\frac{1}{\Gamma(\alpha)} \begin{cases}{[t(1-s)]^{\alpha-1}-(t-s)^{\alpha-1},} & 0 \leq s \leq t \leq 1 \\ {[t(1-s)]^{\alpha-1},} & 0 \leq t \leq s \leq 1\end{cases}
$$

Then

$$
u(t)=\int_{0}^{1} G_{0}(t, s) y(s) d s+t^{\alpha-1} \int_{0}^{1} h(s) u(s) d A(s) .
$$

Multiplying both sides of (2.6) by $h(t)$ and then integrating from 0 to 1 , we have

$$
\begin{aligned}
\int_{0}^{1} h(t) u(t) d A(t)= & \int_{0}^{1} h(t) \int_{0}^{1} G_{0}(t, s) y(s) d s d A(t) \\
& +\int_{0}^{1} t^{\alpha-1} h(t) \int_{0}^{1} h(s) u(s) d A(s) d A(t) .
\end{aligned}
$$

Then, by (2.7) we obtain

$$
\left(1-\int_{0}^{1} t^{\alpha-1} h(t) d A(t)\right) \int_{0}^{1} h(t) u(t) d A(t)=\int_{0}^{1} \int_{0}^{1} h(t) G_{0}(t, s) d A(t) y(s) d s .
$$

Let

$$
\Gamma=\int_{0}^{1} t^{\alpha-1} h(t) d A(t), \quad g_{A}(s)=\int_{0}^{1} G_{0}(t, s) h(t) d A(t) .
$$

From (2.8) and (2.9) we deduce that

$$
\int_{0}^{1} h(t) u(t) d A(t)=\frac{1}{1-\Gamma} \int_{0}^{1} g_{A}(s) y(s) d s .
$$

Substituting (2.10) into (2.6), we obtain

$$
u(t)=\int_{0}^{1} G_{0}(t, s) y(s) d s+\frac{t^{\alpha-1}}{1-\Gamma} \int_{0}^{1} g_{A}(s) y(s) d s=\int_{0}^{1} G(t, s) y(s) d s .
$$

The proof is complete.

Lemma 2.4 Let $0 \leq \Gamma<1$ and $g_{A}(s) \geq 0$ for $s \in[0,1]$. Then the Green function $G(t, s) d e$ fined by (2.2) satisfies

(1) $G:[0,1] \times[0,1] \rightarrow[0, \infty)$ is continuous.

(2) For any $t, s \in[0,1]$, we have $c_{0}(t) \phi(s) \leq G(t, s) \leq \phi(s)$, where

$$
\begin{aligned}
\phi(s) & =\phi_{0}(s)+\frac{g_{A}(s)}{1-\Gamma}, & \phi_{0}(s) & =\frac{\tau(s)^{\alpha-2} s(1-s)^{\alpha-1}}{\Gamma(\alpha-1)}, \\
\tau(s) & =\frac{s}{1-(1-s)^{\frac{\alpha-1}{\alpha-2}}}, & c_{0}(t) & =\min \left\{\frac{(\alpha-1)^{\alpha-1} t^{\alpha-2}(1-t)}{(\alpha-2)^{\alpha-2}}, t^{\alpha-1}\right\} .
\end{aligned}
$$

(3) Then, taking $\theta \in\left(0, \frac{1}{2}\right)$, for any $t \in[\theta, 1-\theta]$ and $s \in[0,1]$, we have $G(t, s) \geq \gamma_{0} \phi(s)$, where $\gamma_{0}=\min _{\theta \leq t \leq 1-\theta} c_{0}(t) \in(0,1)$. 
The proof of Lemma 2.4 is similar to that in [14], so we omit it.

Remark 2.1 By Lemma 2.4 we can obtain that $\frac{\alpha-2}{\alpha-1} \leq \tau(s) \leq 1, \phi_{0}(s) \leq \frac{1}{\alpha-1}$. That is, $\phi(s)$ is bounded, and there exists a constant $\bar{g}>0$ such that $\phi(s) \leq \bar{g}$. It is easy to verify that $\max _{0 \leq t \leq 1} c_{0}(t) \leq 1$.

Let $E=C[0,1]$ and $\|u\|=\sup _{0 \leq t \leq 1}|u(t)|$. Then $(E,\|\cdot\|)$ is a Banach space. Let

$$
P=\{u \in E: u(t) \geq 0, t \in[0,1]\}
$$

and

$$
K=\left\{u \in P: u(t) \geq c_{0}(t)\|u\|, t \in[0,1] ; u(t) \geq \gamma_{0}\|u\|, t \in[\theta, 1-\theta]\right\}
$$

and let $K_{r}=\{u \in K:\|u\|<r\}$. It is easy to see that $K$ is a cone in $E$ and $\overline{K_{R}} \backslash K_{r} \subset K \subset P$.

Throughout the paper, we need the following conditions:

$\left(\mathrm{H}_{1}\right) A:[0,1] \rightarrow \mathbb{R}$ is a function of bounded variation, and $g_{A}(s) \geq 0$ for all $s \in[0,1]$;

$\left(\mathrm{H}_{2}\right) h \in C(0,1) \cap L^{1}(0,1)$, and $0 \leq \Gamma=\int_{0}^{1} t^{\alpha-1} h(s) d A(s)<1$;

$\left(\mathrm{H}_{3}\right) p, q:(0,1) \rightarrow[0, \infty)$ are continuous, $p(t) \not \equiv 0, q(t) \not \equiv 0, t \in[0,1]$, and

$$
\int_{0}^{1} \phi(s) p(s) d s<+\infty, \quad \int_{0}^{1} \phi(s) q(s) d s<+\infty
$$

$\left(\mathrm{H}_{4}\right) f, g:[0,1] \times(0, \infty) \rightarrow[0, \infty)$ are continuous, and for any $0<r<R<+\infty$, $\lim _{m \rightarrow \infty} \sup _{u \in \overline{K_{R}} \backslash K_{r}} \int_{H(m)}(p(s) f(s, u(s))+q(s) g(s, u(s))) d s=0$, where $H(m)=\left[0, \frac{1}{m}\right] \cup$ $\left[\frac{m-1}{m}, 1\right]$.

In what follows, let us define the nonlinear operator $T: \overline{K_{R}} \backslash K_{r} \rightarrow K$ by

$$
(T u)(t)=\int_{0}^{1} G(t, s)[p(s) f(s, u(s))+q(s) g(s, u(s))] d s, \quad t \in[0,1] .
$$

Lemma 2.5 Suppose that $\left(\mathrm{H}_{1}\right)-\left(\mathrm{H}_{4}\right)$ hold. Then $T: \overline{K_{R}} \backslash K_{r} \rightarrow K$ is a completely continuous operator and the fixed point of $T$ in $\overline{K_{R}} \backslash K_{r}$ is the positive solution to BVP (1.1).

Proof It follows from $\left(\mathrm{H}_{4}\right)$ that there exists a natural number $m \geq 2$ such that

$$
\sup _{u \in \overline{K_{R}} \backslash K_{r}} \int_{H(m)} p(s) f(s, u(s))+q(s) g(s, u(s)) d s<1 .
$$

It is easy to see that for any $u \in \overline{K_{R}} \backslash K_{r}$ and $t \in\left[\frac{1}{m}, \frac{m-1}{m}\right]$, we have $\lambda_{0} r \leq u(t) \leq R$, where $\lambda_{0}=\min \left\{c_{0}(t): \frac{1}{m} \leq t \leq \frac{m-1}{m}\right\}>0$. Let

$$
\begin{aligned}
& M_{1}=\max \left\{f(t, x): \frac{1}{m} \leq t \leq \frac{m-1}{m}, \lambda_{0} r \leq x \leq R\right\}, \\
& M_{2}=\max \left\{g(t, x): \frac{1}{m} \leq t \leq \frac{m-1}{m}, \lambda_{0} r \leq x \leq R\right\} .
\end{aligned}
$$


So, by $\left(\mathrm{H}_{3}\right),\left(\mathrm{H}_{4}\right)$, and Remark 2.1 we have

$$
\begin{aligned}
& \sup _{u \in \overline{K_{R}} \backslash K_{r}} \int_{0}^{1} G(t, s)[p(s) f(s, u(s))+q(s) g(s, u(s))] d s \\
& \leq \sup _{u \in \overline{K_{R}} \backslash K_{r}} \int_{0}^{1} \phi(s)[p(s) f(s, u(s))+q(s) g(s, u(s))] d s \\
& \leq \bar{g} \sup _{u \in \overline{K_{R}} \backslash K_{r}} \int_{H(m)} p(s) f(s, u(s))+q(s) g(s, u(s)) d s \\
& \quad+\sup _{u \in \overline{R_{R}} \backslash K_{r}} \int_{\frac{1}{m}}^{\frac{m-1}{m}} \phi(s)[p(s) f(s, u(s))+q(s) g(s, u(s))] d s \\
& \leq \bar{g}+\left(M_{1}+M_{2}\right) \int_{\frac{1}{m}}^{\frac{m-1}{m}} \phi(s)(p(s)+q(s)) d s \\
& \leq \bar{g}+\left(M_{1}+M_{2}\right) \int_{0}^{1} \phi(s)(p(s)+q(s)) d s<+\infty .
\end{aligned}
$$

This implies that the operator $T$ defined by (2.11) is well defined.

Now, we show that $T: \overline{K_{R}} \backslash K_{r} \subset K$. For any $u \in \overline{K_{R}} \backslash K_{r}, t \in[0,1]$, we have

$$
\begin{aligned}
(T u)(t) & =\int_{0}^{1} G(t, s)[p(s) f(s, u(s))+q(s) g(s, u(s))] d s \\
& \leq \int_{0}^{1} \phi(s)[p(s) f(s, u(s))+q(s) g(s, u(s))] d s .
\end{aligned}
$$

Hence,

$$
\|T u\| \leq \int_{0}^{1} \phi(s)[p(s) f(s, u(s))+q(s) g(s, u(s))] d s .
$$

On the other hand, by Lemma 2.4 we have

$$
(T u)(t) \geq c_{0}(t) \int_{0}^{1} \phi(s)[p(s) f(s, u(s))+q(s) g(s, u(s))] d s \geq c_{0}(t)\|T u\|, \quad t \in[0,1],
$$

and at the same time, we can get

$$
(T u)(t) \geq \gamma_{0}\|T u\|, \quad t \in[\theta, 1-\theta] .
$$

Thus, $T u \in K$. Consequently, $T: \overline{K_{R}} \backslash K_{r} \subset K$. Finally, we prove that $T$, which maps $\overline{K_{R}} \backslash K_{r}$ into $K$, is a completely continuous map. Let $D \subset \overline{K_{R}} \backslash K_{r}$ be an arbitrary bounded set. Then from the previous proof we know that $T(D)$ is uniformly bounded.

Next, we show $T(D)$ is equicontinuous. In fact, for any $\epsilon>0$, there exists a natural number $m \geq 2$ such that

$$
\sup _{u \in \overline{K_{R}} \backslash K_{r}} \int_{H(m)} p(s) f(s, u(s))+q(s) g(s, u(s)) d s<\frac{\epsilon}{4 \bar{g}} .
$$


Since $G(t, s)$ is uniformly continuous on $[0,1] \times[0,1]$, for the above $\epsilon>0$, there exists $\delta>0$ such that for any $t_{1}, t_{2} \in[0,1],\left|t_{1}-t_{2}\right|<\delta, s \in\left[\frac{1}{m}, \frac{m-1}{m}\right]$,

$$
\left|G\left(t_{1}, s\right)-G\left(t_{2}, s\right)\right|<\frac{\epsilon}{2\left(\bar{p} M_{1}+\bar{q} M_{2}\right)} .
$$

Consequently, for any $u \in D, t_{1}, t_{2} \in[0,1],\left|t_{1}-t_{2}\right|<\delta$, we have

$$
\begin{aligned}
& \left|(T u)\left(t_{1}\right)-(T u)\left(t_{2}\right)\right| \\
& =\left|\int_{0}^{1}\left(G\left(t_{1}, s\right)-G\left(t_{2}, s\right)\right)(p(s) f(s, u(s))+q(s) g(s, u(s))) d s\right| \\
& \leq 2 \int_{H(m)} \phi(s)[p(s) f(s, u(s))+q(s) g(s, u(s))] d s \\
& \quad+\sup _{u \in D} \int_{\frac{1}{m}}^{\frac{m-1}{m}}\left|\left(G\left(t_{1}, s\right)-G\left(t_{2}, s\right)\right)\right|(p(s) f(s, u(s))+q(s) g(s, u(s))) d s \\
& <2 \bar{g} \frac{\epsilon}{4 \bar{g}}+\frac{\epsilon}{2\left(\bar{p} M_{1}+\bar{q} M_{2}\right)}\left(\bar{p} M_{1}+\bar{q} M_{2}\right) \\
& =\epsilon,
\end{aligned}
$$

where

$$
\bar{p}=\max \left\{p(t): \frac{1}{m} \leq t \leq \frac{m-1}{m}\right\}, \quad \bar{q}=\max \left\{q(t): \frac{1}{m} \leq t \leq \frac{m-1}{m}\right\} .
$$

This shows that $T(D)$ is equicontinuous. By the Arzela-Ascoli theorem, $T: D \rightarrow K$ is compact.

Finally, we prove that $T: \overline{K_{R}} \backslash K_{r} \rightarrow K$ is continuous. Assume that $u_{0}, u_{n} \in \overline{K_{R}} \backslash K_{r}(n=$ $0,1,2, \ldots)$ and $\left\|u_{n}-u_{0}\right\| \rightarrow 0(n \rightarrow \infty)$. Then $r \leq\left\|u_{n}\right\| \leq R$ and $r \leq\left\|u_{0}\right\| \leq R$. For any $\epsilon>0$, by $\left(\mathrm{H}_{4}\right)$ there exists a natural number $m \geq 2$ such that

$$
\sup _{u \in \bar{K}_{R} \backslash K_{r}} \int_{H(m)} p(s) f(s, u(s))+q(s) g(s, u(s)) d s<\frac{\epsilon}{\bar{g}} .
$$

On the other hand, for any $t \in\left[\frac{1}{m}, \frac{m-1}{m}\right]$, we have $\gamma_{0} r \leq u_{n}(t) \leq R(n=0,1,2, \ldots)$. Since $f(t, u), g(t, u)$ are uniformly continuous in $\left[\frac{1}{m}, \frac{m-1}{m}\right] \times\left[\gamma_{0} r, R\right]$, we have that

$$
\begin{aligned}
& \lim _{n \rightarrow \infty}\left|f\left(s, u_{n}(s)\right)-f\left(s, u_{0}(s)\right)\right|=0, \\
& \lim _{n \rightarrow \infty}\left|g\left(s, u_{n}(s)\right)-g\left(s, u_{0}(s)\right)\right|=0
\end{aligned}
$$

uniformly on $\left[\frac{1}{m}, \frac{m-1}{m}\right]$. Then the Lebesgue dominated convergence theorem yields that

$$
\begin{aligned}
& \int_{\frac{1}{m}}^{\frac{m-1}{m}} \phi(s)\left[p(s)\left(f\left(s, u_{n}(s)\right)-f\left(s, u_{0}(s)\right)\right)+q(s)\left(g\left(s, u_{n}(s)\right)-g\left(s, u_{0}(s)\right)\right)\right] d s \rightarrow 0, \\
& \quad n \rightarrow \infty
\end{aligned}
$$


Thus, for the above $\epsilon>0$, there exists a natural number $N$ such that for $n>N$, we have

$$
\int_{\frac{1}{m}}^{\frac{m-1}{m}} \phi(s)\left[p(s)\left(f\left(s, u_{n}(s)\right)-f\left(s, u_{0}(s)\right)\right)+q(s)\left(g\left(s, u_{n}(s)\right)-g\left(s, u_{0}(s)\right)\right)\right] d s<\frac{\epsilon}{2} .
$$

It follows from (2.12), (2.13) that for $n>N$,

$$
\begin{aligned}
\left\|T u_{n}-T u_{0}\right\| \leq & \sup _{u \in \overline{K_{R}} \backslash K_{r}} \int_{H(m)} \phi(s)\left[p(s)\left(f\left(s, u_{n}(s)\right)-f\left(s, u_{0}(s)\right)\right)\right. \\
& \left.+q(s)\left(g\left(s, u_{n}(s)\right)-g\left(s, u_{0}(s)\right)\right)\right] d s \\
& +\sup _{u \in \overline{K_{R}} \backslash K_{r}} \int_{\frac{1}{m}}^{\frac{m-1}{m}} \phi(s)\left[p(s)\left(f\left(s, u_{n}(s)\right)-f\left(s, u_{0}(s)\right)\right)\right. \\
& \left.+q(s)\left(g\left(s, u_{n}(s)\right)-g\left(s, u_{0}(s)\right)\right)\right] d s \\
< & \bar{g} \frac{\epsilon}{2 \bar{g}}+\frac{\epsilon}{2}=\epsilon .
\end{aligned}
$$

This implies that $T: \overline{K_{R}} \backslash K_{r} \rightarrow K$ is continuous. Thus, $T: \overline{K_{R}} \backslash K_{r} \rightarrow K$ is completely continuous. It is clear that if $u$ is a fixed point of $T$ in $\overline{K_{R}} \backslash K_{r}$, then $u$ satisfies (1.1) and is a positive solution of BVP (1.1).

To prove the main results, we need the following well-known fixed point theorem.

Lemma 2.6 [15] Let $P$ be a positive cone in a Banach space $E, \Omega_{1}$ and $\Omega_{2}$ be two bounded open sets in $E$ such that $\theta \in \Omega_{1}$ and $\overline{\Omega_{1}} \subset \Omega_{2}, A: P \cap\left(\overline{\Omega_{2}} \backslash \Omega_{1}\right) \rightarrow P$ be a completely continuous operator, where $\theta$ denotes the zero element of $E$. Suppose that one of the following two conditions holds:

(i) $\|A u\| \leq\|u\|, \forall u \in P \cap \partial \Omega_{1} ;\|A u\| \geq\|u\|, \forall u \in P \cap \partial \Omega_{2}$;

(ii) $\|A u\| \geq\|u\|, \forall u \in P \cap \partial \Omega_{1} ;\|A u\| \leq\|u\|, \forall u \in P \cap \partial \Omega_{2}$.

Then $A$ has a fixed point in $P \cap\left(\overline{\Omega_{2}} \backslash \Omega_{1}\right)$.

\section{Existence of a positive solution}

In the following, for convenience, we set

$$
\begin{aligned}
& \bar{f}_{0}=\limsup _{x \rightarrow 0} \max _{t \in[0,1]} \frac{f(t, x)}{x}, \quad \bar{g}_{0}=\limsup _{x \rightarrow 0} \max _{t \in[0,1]} \frac{g(t, x)}{x}, \\
& f_{-0}=\liminf _{x \rightarrow 0} \min _{t \in[\theta, 1-\theta]} \frac{f(t, x)}{x}, \quad \underline{g}_{0}=\liminf _{x \rightarrow 0} \min _{t \in[\theta, 1-\theta]} \frac{g(t, x)}{x}, \\
& \bar{f}_{\infty}=\limsup _{x \rightarrow \infty} \max _{t \in[0,1]} \frac{f(t, x)}{x}, \quad \bar{g}_{\infty}=\limsup _{x \rightarrow \infty} \max _{t \in[0,1]} \frac{g(t, x)}{x}, \\
& f_{-\infty}=\liminf _{x \rightarrow \infty} \min _{t \in[\theta, 1-\theta]} \frac{f(t, x)}{x}, \quad \underline{g}_{\infty}=\liminf _{x \rightarrow \infty} \min _{t \in[\theta, 1-\theta]} \frac{g(t, x)}{x}, \\
& m_{0}=\left[\int_{0}^{1} \phi(s)[p(s)+q(s)] d s\right]^{-1}, \quad m_{1}=\left[\gamma_{0} \int_{\theta}^{1-\theta} \phi(s) p(s) d s\right]^{-1}, \\
& m_{2}=\left[\gamma_{0} \int_{\theta}^{1-\theta} \phi(s) q(s) d s\right]^{-1} .
\end{aligned}
$$


Theorem 3.1 Let $f, g:[0,1] \times[0,+\infty) \rightarrow[0,+\infty)$ be continuous. Assume that $\left(\mathrm{H}_{1}\right)-\left(\mathrm{H}_{3}\right)$ and $\bar{f}_{0}<m_{0}, \bar{g}_{0}<m_{0}$ hold and one of the two conditions $f_{-\infty}>m_{1}$ and $\underline{g}_{\infty}>m_{2}$ is satisfied. Then BVP (1.1) has at least one positive solution.

Proof First, we consider the case $f_{-\infty}>m_{1}$. Since $\bar{f}_{0}<m_{0}, \bar{g}_{0}<m_{0}$, we can choose $\epsilon_{0}>0$ such that $m_{0}-\epsilon_{0}>0$ and $\bar{f}_{0}<m_{0}-\epsilon_{0}, \bar{g}_{0}<m_{0}-\epsilon_{0}$, and also there exists $r_{0}>0$ such that for any $0<x \leq r_{0}, 0 \leq t \leq 1$, we have

$$
f(t, x) \leq\left(m_{0}-\epsilon_{0}\right) x, \quad g(t, x) \leq\left(m_{0}-\epsilon_{0}\right) x .
$$

Set $\Omega_{0}=\left\{u \in E:\|u\|<r_{0}\right\}$. We can see that $\Omega_{0}$ is a bounded open subset in $E$, and for any $u \in \partial \Omega_{0} \cap K, 0<u(t) \leq r_{0}, t \in[0,1]$, from expression (3.1) we deduce the following inequalities:

$$
\begin{aligned}
\|T u\| & =\sup _{t \in[0,1]}\left|\int_{0}^{1} G(t, s)[p(s) f(s, u(s))+q(s) g(s, u(s))] d s\right| \\
& \leq\left(m_{0}-\epsilon_{0}\right) \sup _{t \in[0,1]}\left|\int_{0}^{1} G(t, s)[p(s)+q(s)] u(s) d s\right| \\
& \leq\left(m_{0}-\epsilon_{0}\right) \sup _{t \in[0,1]}\left|\int_{0}^{1} G(t, s)[p(s)+q(s)] d s\right|\|u\| \\
& \leq m_{0} \int_{0}^{1} \phi(s)[p(s)+q(s)] d s\|u\|=\|u\| .
\end{aligned}
$$

Consequently,

$$
\|T u\| \leq\|u\|, \quad \forall u \in \partial \Omega_{0} \cap K .
$$

On the other hand, since $f_{-\infty}>m_{1}$, we can choose $\epsilon_{1}>0$ such that $f_{-\infty}>\left(m_{1}+\epsilon_{1}\right)$, and also there exists $R_{1}>r_{0}>0$ such that for any $x \geq R_{1}, t \in[\theta, 1-\theta]$, we have

$$
f(t, x) \geq\left(m_{1}+\epsilon_{1}\right) x
$$

Let $\overline{R_{1}}=\frac{R_{1}}{\gamma_{0}}$ and $\Omega_{1}=\left\{u \in E:\|u\|<\overline{R_{1}}\right\}$. It is easy to see that $\Omega_{1}$ is a bounded open subset in $E$, and from the expression (3.3) we have that for any $u \in \partial \Omega_{1} \cap K, u(t) \geq \gamma_{0}\|u\|=R_{1}$, $t \in[\theta, 1-\theta]$,

$$
\begin{aligned}
\|T u\| & =\sup _{t \in[0,1]}\left|\int_{0}^{1} G(t, s)[p(s) f(s, u(s))+q(s) g(s, u(s))] d s\right| \\
& \geq \int_{\theta}^{1-\theta} G(t, s) p(s) f(s, u(s)) d s \\
& \geq \gamma_{0}\left(m_{1}+\epsilon_{1}\right) \int_{\theta}^{1-\theta} \phi(s) p(s) d s\|u\| \\
& \geq \gamma_{0} m_{1} \int_{\theta}^{1-\theta} \phi(s) p(s) d s\|u\|=\|u\| .
\end{aligned}
$$

Consequently,

$$
\|T u\| \geq\|u\|, \quad \forall u \in \partial \Omega_{1} \cap K .
$$


Applying Lemma 2.6 to (3.2) and (3.4) yields that $T$ has a fixed point $u^{*} \in K \cap\left(\overline{\Omega_{1}} \backslash \Omega_{0}\right)$, and hence $u^{*}$ is a positive solution of BVP (1.1).

In a similar way, when the case that $\underline{g}_{\infty}>m_{2}$ holds, we can prove that the conclusion of Theorem 3.1 also holds. The proof of Theorem 3.1 is completed.

Theorem 3.2 Assume that $\left(\mathrm{H}_{1}\right)-\left(\mathrm{H}_{4}\right)$ and $\bar{f}_{\infty}<m_{0}, \bar{g}_{\infty}<m_{0}$ hold. Then BVP (1.1) has at least one positive solution.

The proof is similar to the previous one, so we omit it.

\section{Existence of multiple positive solutions}

In this section, we discuss the existence of multiple positive solutions of BVP (1.1). We obtain the following existence theorems.

Theorem 4.1 Assume that $\left(\mathrm{H}_{1}\right)-\left(\mathrm{H}_{4}\right)$ hold and $f_{-\infty}>m_{1}$ are satisfied. Further, assume that the following condition holds:

$$
(*) \quad \int_{0}^{1} p(s) f(s, u(s))+q(s) g(s, u(s)) d s<\frac{1}{\bar{g}}, \quad \forall u \in \partial \Omega \cap K,
$$

where $\phi(s) \leq \bar{g}, \partial \Omega=\{u \in E:\|u\|=1\}$. Then BVP (1.1) has at least two positive solutions.

Proof Since $\underline{g}_{0}=+\infty>m_{2}$, there exist $\epsilon_{2}>0,0<r_{1}<1$ such that for any $0<x \leq r_{1}, \theta \leq$ $t \leq 1-\theta$, we have

$$
g(t, x) \geq\left(m_{2}+\epsilon_{2}\right) x
$$

Set $\Omega_{2}=\left\{u \in E:\|u\|<r_{1}\right\}$. Then $\Omega_{2}$ is a bounded open subset in $E$, and for any $u \in \partial \Omega_{2} \cap K$ and $0<u(t) \leq r_{1}$, by expression (4.1) we have

$$
\begin{aligned}
\|T u\| & =\sup _{t \in[0,1]}\left|\int_{0}^{1} G(t, s)[p(s) f(s, u(s))+q(s) g(s, u(s))] d s\right| \\
& \geq \int_{\theta}^{1-\theta} G(t, s) q(s) g(s, u(s)) d s \\
& \geq \gamma_{0}\left(m_{2}+\epsilon_{2}\right) \int_{\theta}^{1-\theta} \phi(s) q(s) d s\|u\| \\
& \geq \gamma_{0} m_{2} \int_{\theta}^{1-\theta} \phi(s) q(s) d s\|u\|=\|u\| .
\end{aligned}
$$

Consequently,

$$
\|T u\| \geq\|u\|, \quad \forall u \in \partial \Omega_{2} \cap K
$$

Since $f_{-\infty}>m_{1}$, there exist $\epsilon_{3}>0, R_{2}>1$ such that for any $x \geq R_{2}, \theta \leq t \leq 1-\theta$, we have

$$
f(t, x) \geq\left(m_{1}+\epsilon_{3}\right) x
$$


Let $R_{3}=\frac{R_{2}}{\gamma_{0}}$ and $\Omega_{3}=\left\{u \in E:\|u\|<R_{3}\right\}$. Then $\Omega_{3}$ is a bounded open subset in $E$, and so for any $u \in \partial \Omega_{3} \cap K$ and $u(t) \geq \gamma_{0}\|u\|=R_{2}, t \in[\theta, 1-\theta]$, by expression (4.3) we have

$$
\begin{aligned}
\|T u\| & =\sup _{t \in[0,1]}\left|\int_{0}^{1} G(t, s)[p(s) f(s, u(s))+q(s) g(s, u(s))] d s\right| \\
& \geq \int_{\theta}^{1-\theta} G(t, s) p(s) f(s, u(s)) d s \\
& \geq \gamma_{0}\left(m_{1}+\epsilon_{3}\right) \int_{\theta}^{1-\theta} \phi(s) p(s) d s\|u\| \\
& \geq \gamma_{0} m_{1} \int_{\theta}^{1-\theta} \phi(s) p(s) d s\|u\|=\|u\| .
\end{aligned}
$$

Consequently,

$$
\|T u\| \geq\|u\|, \quad \forall u \in \partial \Omega_{3} \cap K .
$$

On the other hand, by $(*)$ we have for any $u \in \partial \Omega \cap K, t \in[0,1]$,

$$
\begin{aligned}
\|T u\| & =\sup _{t \in[0,1]}\left|\int_{0}^{1} G(t, s)[p(s) f(s, u(s))+q(s) g(s, u(s))] d s\right| \\
& \leq \int_{0}^{1} \phi(s)[p(s) f(s, u(s))+q(s) g(s, u(s))] d s \\
& \leq \bar{g} \int_{0}^{1}[p(s) f(s, u(s))+q(s) g(s, u(s))] d s \leq \bar{g} \frac{1}{\bar{g}}=1=\|u\| .
\end{aligned}
$$

Consequently,

$$
\|T u\| \leq\|u\|, \quad \forall u \in \partial \Omega \cap K .
$$

Therefore, from (4.2), (4.5), and Lemma 2.6 it follows that BVP (1.1) has a positive solution $u_{0} \in K$ with $r_{1} \leq\|u\|<1$. In the same way, from (4.4), (4.5), and Lemma 2.6 it follows that BVP (1.1) has a positive solution $u_{1} \in K$ with $1<\|u\| \leq R_{3}$. Therefore, BVP (1.1) has at least two positive solutions $u_{0}, u_{1} \in K$.

Theorem 4.2 Assume that $\left(\mathrm{H}_{1}\right)-\left(\mathrm{H}_{4}\right)$ hold and $\underline{g}_{\infty}>m_{2}$ are satisfied. Further, assume that the following condition holds:

$$
\int_{0}^{1} p(s) f(s, u(s))+q(s) g(s, u(s)) d s<\frac{1}{\bar{g}}, \quad \forall u \in \partial \Omega \cap K,
$$

where $\phi(s) \leq \bar{g}, \partial \Omega=\{u \in E:\|u\|=1\}$. Then BVP (1.1) has at least two positive solutions.

The proof is similar to the previous one, so we omit it. 


\section{Example}

Example 5.1 We consider BVP (1.1) with $\alpha=\frac{7}{2}, p(t)=q(t)=\frac{1}{200 \sqrt{1-t^{2}}}, h(t)=t^{-\frac{1}{2}}, f(t, x)=$ $5-t+|\ln x|+x^{\frac{3}{2}}, g(t, x)=2-t+|\ln x|$, and

$$
A(t)= \begin{cases}0, & t \in\left[0, \frac{1}{2}\right) \\ 2, & t \in\left[\frac{1}{2}, 1\right]\end{cases}
$$

Thus, $\Gamma=\int_{0}^{1} t^{\frac{5}{2}} t^{-\frac{1}{2}} d A(t)=2 \times\left(\frac{1}{2}\right)^{2}=\frac{1}{2}$. Obviously, $g_{A}(s) \leq 1$ and $\phi(s)=\phi_{0}(s)+\frac{g_{A}(s)}{1-\Gamma} \leq 4=\bar{g}$. We define the cone

$$
K=\left\{u \in C[0,1]: u(t) \geq c_{0}(t)\|u\|, t \in[0,1] ; u(t) \geq \gamma_{0}\|u\|, t \in[\theta, 1-\theta]\right\},
$$

in which

$$
c_{0}(t)=\min \left\{\frac{\left(\frac{5}{2}\right)^{\frac{5}{2}} t^{\frac{3}{2}}(1-t)}{\frac{3}{2}^{\frac{3}{2}}}, t^{\frac{5}{2}}\right\} .
$$

For any $0<r<R<+\infty$ and $u \in \overline{K_{R}} \backslash K_{r}$, we have $u(t) \geq c_{0}(t)\|u\|, t \in[0,1]$. Then $0 \leq r c_{0}(t) \leq$ $u(t) \leq R, t \in[0,1]$. Since $|\ln x|$ is decreasing on $(0,1)$ and is increasing on $(1,+\infty)$, we have

$$
|\ln u(x)| \leq\left|\ln r c_{0}(t)\right|+|\ln R| \leq|\ln r|+|\ln R|+\left|\ln c_{0}(t)\right|
$$

It is easy to verify that $\frac{\left|\ln c_{0}(t)\right|}{200 \sqrt{1-t^{2}}} \in L^{1}(0,1)$. The absolute continuity of the integral yields that

$$
\lim _{m \rightarrow \infty} \int_{H(m)} \frac{\left|\ln c_{0}(s)\right|}{200 \sqrt{1-s^{2}}} d s=0
$$

So, by (5.1) and (5.2) we get

$$
\begin{aligned}
& \sup _{u \in \bar{K}_{R} \backslash K_{r}} \int_{H(m)} \frac{1}{200 \sqrt{1-s^{2}}}\left[2-s+|\ln u(s)|+5-s+|\ln u(s)|+(u(s))^{\frac{3}{2}}\right] d s \\
& \leq \int_{H(m)} \frac{1}{200 \sqrt{1-s^{2}}}\left[7-2 s+2|\ln r|+2|\ln R|+2\left|\ln c_{0}(s)\right|+R^{\frac{3}{2}}\right] d s \\
& \leq \\
& \quad\left(8+R^{\frac{3}{2}}+4|\ln r|+4|\ln R|\right) \int_{H(m)} \frac{1}{200 \sqrt{1-s^{2}}} d s+\int_{H(m)} \frac{1}{100 \sqrt{1-s^{2}}}\left|\ln c_{0}(s)\right| d s \\
& \quad \frac{8+R^{\frac{3}{2}}+4|\ln r|+4|\ln R|}{200}\left[\arcsin \frac{1}{m}+\arcsin 1-\arcsin \left(1-\frac{1}{m}\right)\right] \\
& \quad+\int_{H(m)} \frac{1}{100 \sqrt{1-s^{2}}}\left|\ln c_{0}(s)\right| d s .
\end{aligned}
$$

By (5.2) we know that, as $m \rightarrow \infty$, the limit of (5.3) is 0 . On the other hand, by calculation we have

$$
\begin{aligned}
& \underline{g}_{0}=\liminf _{x \rightarrow 0} \min _{t \in[\theta, 1-\theta]} \frac{g(t, x)}{x}=\liminf _{x \rightarrow 0} \frac{1+\theta+|\ln x|}{x}=\infty>m_{2}, \\
& f_{-\infty}=\liminf _{x \rightarrow \infty} \min _{t \in[\theta, 1-\theta]} \frac{f(t, x)}{x}=\liminf _{x \rightarrow \infty} \frac{4+\theta+|\ln x|}{x}+x^{\frac{1}{2}}=\infty>m_{1},
\end{aligned}
$$


where

$$
m_{1}=m_{2}=\left[\gamma_{0} \int_{\theta}^{1-\theta} \frac{\phi(s)}{200 \sqrt{1-s^{2}}} d s\right]^{-1}
$$

with $\gamma_{0}=\min _{\theta \leq t \leq 1-\theta} c_{0}(t)$. On the other hand, for any $u \in \partial \Omega \cap K, t \in[0,1]$, we have

$$
\begin{aligned}
f(t, u(t))+g(t, u(t)) & =7-2 t+2|\ln u(t)|+u(t)^{\frac{3}{2}} \\
& \leq 8+2\left|\ln c_{0}(t)\right| .
\end{aligned}
$$

So we obtain

$$
\begin{aligned}
\int_{0}^{1} p(s) f(s, u(s))+q(s) g(s, u(s)) d s & =\int_{0}^{1} \frac{1}{200 \sqrt{1-s^{2}}}[f(s, u(s))+g(s, u(s))] d s \\
& \leq \int_{0}^{1} \frac{1}{200 \sqrt{1-s^{2}}}\left[8+2\left|\ln c_{0}(s)\right|\right] d s \\
& =\frac{\pi}{50}+\frac{1}{100} \int_{0}^{1} \frac{\left|\ln c_{0}(s)\right|}{\sqrt{1-s^{2}}} d s \\
& \leq \frac{\pi}{50}+\frac{1}{10} \\
& <\frac{1}{4} .
\end{aligned}
$$

Therefore, the assumptions of Theorem 4.1 are satisfied. Thus the problem considered possesses two positive solutions in $K$.

Competing interests

The authors declare that there is no conflict of interests regarding the publication of this paper.

\section{Authors' contributions}

All authors contributed equally and significantly in writing this article. All authors read and approved the final manuscript.

\section{Acknowledgements}

The authors would like to thank the referee for his very important comments that improved the results and the quality of the paper. The authors were supported financially by the National Natural Science Foundation of China (11371221, 11571296), the Specialized Research Foundation for the Doctoral Program of Higher Education of China (20123705110001), and the Program for Scientific Research Innovation Team in Colleges and Universities of Shandong Province.

Received: 26 August 2015 Accepted: 23 November 2015 Published online: 09 December 2015

\section{References}

1. Kilbas, AA, Srivastava, HM, Trujillo, JJ: Theory and Applications of Fractional Differential Equations. Elsevier, Amsterdam (2006)

2. Lazarevic, MP, Spasic, AM: Finite-time stability analysis of fractional order time-delay systems: Gronwall's approach Math. Comput. Model. 49, 475-481 (2009)

3. Delbosco, D: Fractional calculus and function spaces. J. Fract. Calc. 6, 45-53 (1994)

4. Cabada, A, Wang, G: Positive solutions of nonlinear fractional differential equations with integral boundary value conditions. J. Math. Anal. Appl. 389, 403-411 (2012)

5. Podlubny, I: Fractional Differential Equations. Mathematics in Science and Engineering, vol. 198. Academic Press, New York (1999)

6. Kilbas, AA, Srivastava, HM, Trujillo, JJ: Theory and Applications of Fractional Differential Equations. North-Holland Mathematics Studies, vol. 204. Elsevier, Amsterdam (2006)

7. Cabada, A, Hamdi, Z: Nonlinear fractional differential equations with integral boundary value conditions. Appl. Math. Comput. 228, 251-257 (2014)

8. Sun, Y, Zhao, M: Positive solutions for a class of fractional differential equations with integral boundary conditions. Appl. Math. Lett. 34, 17-21 (2014) 
9. Zhang, X, Wang, L, Sun, Q: Existence of positive solutions for a class of nonlinear fractional differential equations with integral boundary conditions and a parameter. Appl. Math. Comput. 226, 708-718 (2014)

10. Wang, Y, Liu, L, Wu, Y: Positive solutions for a nonlocal fractional differential equation. Nonlinear Anal. 74, 3599-3605 (2011)

11. Zhao, Y, Chen, H, Huang, L: Existence of positive solutions for nonlinear fractional functional differential equation. Comput. Math. Appl. 64, 3456-3467 (2012)

12. Lomtatidze, A, Malaguti, L: On a nonlocal boundary-value problems for second order nonlinear singular differential equations. Georgian Math. J. 7, 133-154 (2000)

13. Samko, SG, Kilbas, AA, Marichev, Ol: Fractional Integral and Derivative: Theory and Applications. Gordon \& Breach, Yverdon (1993)

14. Hao, $X$, Liu, L, Wu, Y, Sun, Q: Positive solutions for nonlinear $n$ th-order singular eigenvalue problem with nonlocal conditions. Nonlinear Anal. 73, 1653-1662 (2010)

15. Guo, D, Lakshmikantham, V: Nonlinear Problems in Abstract Cones. Academic Press, San Diego (1988)

Submit your manuscript to a SpringerOpen ${ }^{\circ}$ journal and benefit from:

- Convenient online submission

Rigorous peer review

- Immediate publication on acceptance

- Open access: articles freely available online

- High visibility within the field

- Retaining the copyright to your article 